\title{
Synthesis of Some Novel Aromatic Alkynyl Silanes: Mesomorphic Characterization of Ethynyl- Substituted Rod-Shaped Molecules
}

\section{H. T. Srinivasa \& S. Hariprasad}

To cite this article: H. T. Srinivasa \& S. Hariprasad (2014) Synthesis of Some Novel Aromatic Alkynyl Silanes: Mesomorphic Characterization of Ethynyl-Substituted Rod-Shaped Molecules, Molecular Crystals and Liquid Crystals, 588:1, 17-27, DOI: 10.1080/15421406.2013.822299

To link to this article: http://dx.doi.org/10.1080/15421406.2013.822299

+ View supplementary material $\widetilde{T}$

Submit your article to this journal ¿

山 Article views: 137

View related articles

Citing articles: 1 View citing articles ¿ 


\title{
Synthesis of Some Novel Aromatic Alkynyl Silanes: Mesomorphic Characterization of Ethynyl-Substituted Rod-Shaped Molecules
}

\author{
H. T. SRINIVASA ${ }^{1,2}$ AND S. HARIPRASAD ${ }^{1, *}$ \\ ${ }^{1}$ Department of Studies in Chemistry, Bangalore University, Bangalore, India \\ ${ }^{2}$ Raman Research Institute, Sadashivanagara, Bangalore, India
}

\begin{abstract}
The synthesis and characterization of 10 new rod-shaped substituted benzoates possessing the 4-(2-trimethylsilyl)ethynyl group in terminal phenyl position is reported employing the simple and efficient Sonogashira cross-coupling and DCC esterification. 4'-Dodecylbiphenyl-4-carboxylic(2-trimethylsilylethynyl)-phenyl ester (3i), 4-(4'hexadecyloxybenzyloxy) carboxylic-4-trimethylsilanylethynyl-phenyl ester (3j), and 1,4bis(4-(pentyloxy)phenyl)buta-1,3-diyne (7) compounds were evaluated for liquid crystal property.
\end{abstract}

[Supplemental materials are available for this article. Go to the publisher's online edition of Molecular Crystals and Liquid Crystals to view the free supplemental file.]

Keywords Liquid crystals; Smectic mesophase; Sonogashira coupling; 2-trimethylsilylethynyl

\section{Introduction}

Liquid crystals form an important class in material chemistry. In particular, liquid crystals possessing the trimethylsilyl- acetylenic group have gained importance in the field of synthetic chemistry such as for masking anionic synthons to prepare metalcoordinated complexes [1,2]. The ethynyl silanes have also been used to prepare many interesting classes of polycyclic aromatic compounds [3]. Very few reports are found in the literature on molecular structure containing trimethylsilyl- acetylene moiety functionalized in material science. Metallomesogens of platinum metal-carbon sigma bond having di(arylethynyl)bis(trimethylphosphine)platinum(II) complex [4] and $\beta$-Dgalactopyranoside end-groups containing chiral amphiphilic mesogenic materials with selfassembled nanostructures compounds have been reported [5]. Liquid crystals consisting of a rigid and linear molecular framework with extended $\pi$-conjugated electronic structure of difluoroterphenyl core with short and bulky trimethylsilyl terminal groups provide stability to the mesophase [6]. The pyrimidinylphenyldiacetylenes [7], azotolanes [8,9], 1,4bis(phenylethynyl)benzene [10], 1,4-bis(2-naphthyl)-1,3-butadiynes [11], and high birefringence property having diphenyl-diacetylene-based nematogens [12] are also reported. Alkyl-substituted oligo( $p$-phenylene)ethynylenes [13] and 1,2-(4,4-dialkoxyaryl)acetylene

*Address correspondence to S. Hariprasad, Department of Studies in Chemistry, Bangalore University, Central College Campus, Bangalore 560001, India. Tel.: +918022961351; Fax: +918022961331. E-mail: hariprasad@bub.ernet.in 
with solid polymorphism [14, 15] and mesomorphism showing triptycene derivatives [16] have been achieved using different synthetic routes and in the presence of different concentrations of palladium catalysts. The reported synthetic strategies used for the preparation of trimethylsilylethynyl containing biological molecules and rod, disc, and banana-shaped compounds suffer from certain synthetic procedures [17-19]. Recently, Hanasaki et al. and Liao et al. reported polar groups containing symmetric and achiral swallow tail-derived siloxane dimers [20,21].

Our research laboratory focuses on the synthesis and reactions of novel organosilicon reagents [22-27]. In continuation of our studies and in the light of the importance of the aforesaid work in literature, it was in our interest to attempt the preparation and study of liquid crystal properties of some low molar mass silyl- substituted molecules [28].

In this article, we report the simple and efficient synthesis of some 4-(2trimethylsilyl)ethynyl phenyl ester derivatives using Sonogashira cross-coupling reaction and DCC esterification to obtain alkynyl aromatics. Their mesomorphic studies are reported.

\section{Experimental}

\section{Materials and Characterization}

All chemicals were purchased from commercial sources (Aldrich, Merck, and Fluka, Bangalore, Karnataka, India). Triethylamine and $N, N$-dimethylformamide were distilled and stored on molecular sieves before use. IR spectra were recorded on Shimadzu FTIR8400 spectrophotometer. ${ }^{1} \mathrm{H}-\mathrm{NMR}$ and ${ }^{13} \mathrm{C}-\mathrm{NMR}$ spectra were recorded in $\mathrm{CDCl}_{3}$ with a Bruker AMX $400 \mathrm{MHz}$ and a Bruker AMX $100 \mathrm{MHz}$ spectrometer, respectively, using tetramethylsilane as an internal standard. Mass spectral analysis was carried on a Shimadzu GC-MS QP5050A instrument equipped with a $30 \mathrm{~m} \times 0.32 \mathrm{~mm}$ BP-5 capillary column. Elemental analysis was carried out by a Carlo-Erba 1106 analyzer. Stability was checked by a Perkin-Elmer TGA 4000 analyzer. Melting points, thermal behavior, and enthalpies were examined using a combination of differential scanning calorimetry (DSC) heating from room temperature to isotropic temperatures at the scan rate of $5^{\circ} \mathrm{C} / \mathrm{min}$ (Perkin-Elmer Model Pyris 1D) and polarizing optical microscopy (POM) using Olympus BX50 microscope equipped with a heating hot stage Mettler FP82HT and a central processor Mettler FP80. Melting points remain uncorrected. X-ray diffraction (XRD) studies were carried out on unoriented samples using $\mathrm{Cu}-\mathrm{K} \alpha(\lambda=1.54 \AA)$ radiation from a Rigaku Ultrax 18 rotating anode generator $(5.4 \mathrm{~kW})$ monochromated with a graphite crystal. The samples were held in sealed Lindemann capillary tubes $(0.7 \mathrm{~mm}$ diameter $)$ and the diffraction patterns were collected on a two-dimensional Marresearch image plate.

Synthesis and Analytical Data. All benzoic acid derivatives were prepared according to the reported literature procedures $[6,29,30] .1-\left(4^{\prime} ;-\right.$ Hydroxyphenyl)-2-trimethylsilylacetylene (2) [20] was used to prepare the compounds 3a-j. 1-(4';-n-Pentyloxyphenyl)-2trimethylsilylacetylene (5) was used for the preparation of compounds $\mathbf{7}$ and $\mathbf{8}$, respectively, according to Scheme 1.

All chemical structures are shown in Table 1.

Reagents: a) Ethynyltrimethylsilane, $\mathrm{PdCl}_{2}$, TPP, $\mathrm{NEt}_{3}, \mathrm{Cu}(\mathrm{OAc})_{2}, 80^{\circ} \mathrm{C}, 24$ h. b) Benzoic acids, DCC, DMAP, dry $\mathrm{CH}_{2} \mathrm{Cl}_{2}, 45$ min. c) $n-\mathrm{C}_{5} \mathrm{H}_{11} \mathrm{Br}, \mathrm{K}_{2} \mathrm{CO}_{3}, 85^{\circ} \mathrm{C}$, methylethylketone. d) $2 \%$ aqueous $\mathrm{K}_{2} \mathrm{CO}_{3}, \mathrm{MEOH}$, rt. e) $\mathrm{Cu}(\mathrm{OAc})_{2}$, methanol/pyridine, reflux, $24 \mathrm{~h}$. f) $5 \% \mathrm{Pd}-\mathrm{C}, \mathrm{H}_{2}, 1,4$-dioxane, $60^{\circ} \mathrm{C}, 12 \mathrm{~h}$. 


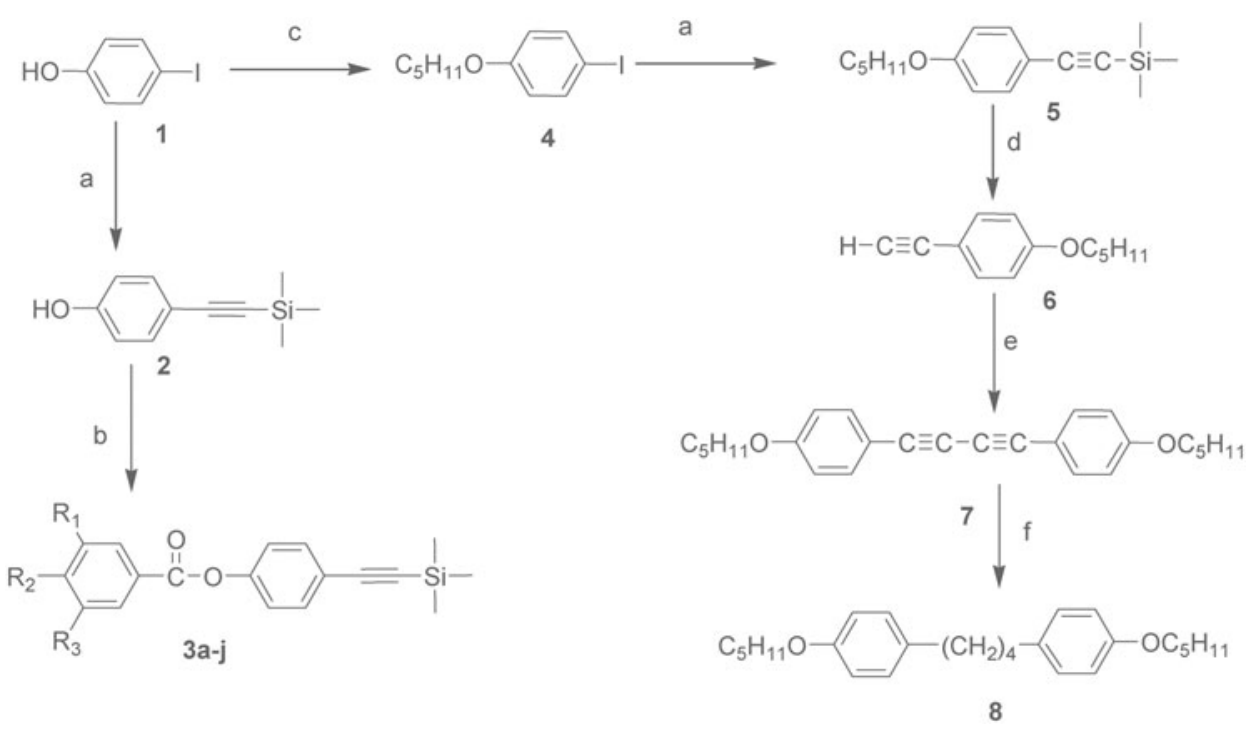

Scheme 1. General synthetic method used for the preparation of substituted 4(trimethylsilyl)ethynylbenzoates (3a-j), 1,4-bis[4-(n-pentyloxy)phenyl]buta-1,3-diyne (7), and 1,4-bis[4(pentyloxy)phenyl]butane (8).

Preparation of 1-(4'-Hydroxyphenyl)-2-trimethylsilylacetylene (2) [20]. To a refluxing suspension of iodophenol $(2.5 \mathrm{~g}, 1.0 \mathrm{mmol})$, copper acetate monohydrate $(1.35 \mathrm{~g}$, $1.07 \mathrm{mmol})$, and palladium chloride $(1.8 \mathrm{~g}, 1.07 \mathrm{mmol})$ in triethylamine $(10 \mathrm{~mL})$ in an inert atmosphere was added dropwise trimethylsilylacetylene (1.36 g, $1.20 \mathrm{mmol}$ ) over $30 \mathrm{~min}$. The reaction mixture was further refluxed at $80^{\circ} \mathrm{C}$ for $24 \mathrm{~h}$ under nitrogen atmosphere and monitored by TLC for completion of reaction. The mixture was cooled and filtered to remove the solids. The volatile solvents were evaporated under reduced pressure, and the resulting crude light brown colored viscous oil was extracted into ether, washed with saturated potassium bicarbonate solution, dried over anhydrous sodium sulfate, and concentrated. The silylated product was purified by column chromatography on silica gel with $3 \%$ ethyl acetate in hexane as eluent. Yield $=1.58 \mathrm{~g}(79 \%)$.

\section{General Procedure for the Preparation of 4-(2-trimethylsilylethynyl) phenylesters (3a-j)}

A mixture of 4-(2-(trimethylsilyl)ethynyl)phenol (1 mmol), benzoic acid derivatives (1 mmol), $N, N$-dicyclohexylcarbodiimide (DCC) $(1.2 \mathrm{mmol})$, and catalytic quantity of $N, N$ dimethylaminopyrimidine (DMAP) in anhydrous dichloromethane $(10 \mathrm{~mL})$ was stirred at room temperature for $45 \mathrm{~min}$. After completion of reaction, the $N, N$-dicyclohexylurea formed was filtered off and the filtrate diluted with dichloromethane. This solution was washed successively with water $(2 \times 30 \mathrm{~mL}), 5 \%$ aqueous sodium hydroxide solution $(2 \times$ $25 \mathrm{~mL})$, water $(2 \times 25 \mathrm{~mL})$, and dried over anhydrous sodium sulfate. The crude product was chromatographed on silica gel (100-200 mesh size) using 2\% ethyl acetate in petroleum ether as an eluent. Removal of solvent afforded white solid, which was recrystallized from hexane.

4'-(2-Trimethylsilylethynyl)phenyl-4-cyanobenzoate (3a). IR: 2926, 2821, 2233, 2160, 1747, 1597, 1502, 1454, 1404, 1257, 1195, 1066, 1016, 761, $686 \mathrm{~cm}^{-1}$; ${ }^{1} \mathrm{H}$ NMR $\left(\mathrm{ppm}, \mathrm{CDCl}_{3}\right): 8.2(\mathrm{~d}, 2 \mathrm{H}, J=2.1 \mathrm{~Hz}), 7.8(\mathrm{~d}, 2 \mathrm{H}, J=2.0 \mathrm{~Hz}), 7.5(\mathrm{~d}, 2 \mathrm{H}, J=1.9 \mathrm{~Hz})$, 
Table 1. Chemical structures and melting points (in ${ }^{\circ} \mathrm{C}$ ) of compounds $\mathbf{3 a}-\mathbf{j}$

\begin{tabular}{|c|c|c|}
\hline Compound & Chemical Structure & Melting Points \\
\hline $\mathbf{3 a}$ & & $137-139$ \\
\hline $\mathbf{3 b}$ & & $86-88$ \\
\hline $3 \mathrm{c}$ & & $126-128$ \\
\hline $3 d$ & & $165-167$ \\
\hline $3 \mathbf{e}$ & & Viscous liquid \\
\hline 3f & & $50-52$ \\
\hline $3 g$ & & $84-85$ \\
\hline $3 \mathbf{h}$ & & $70-72$ \\
\hline $3 \mathbf{i}$ & & 90-92 \\
\hline $3 \mathbf{j}$ & & $102-104$ \\
\hline
\end{tabular}

$7.1(\mathrm{~d}, 2 \mathrm{H}, J=1.8 \mathrm{~Hz}), 0.26(\mathrm{~s}, 9 \mathrm{H}) ;{ }^{13} \mathrm{C} \mathrm{NMR}\left(\mathrm{ppm}, \mathrm{CDCl}_{3}\right): 164.6,159.7,150.9,133.1$, 130.5, 129.6, 122.6, 121.6, 120.3, 114.5, 104.2, 94.3, 55.5, 0.02; GC-MS: $319.8(\mathrm{~m} / \mathrm{e}$, relative intensity), 318.8 (4.02), 304.9 (0.96), 303.8 (3.17), 276.2 (0.59), 174 (3.29), 146 (6.65), 130 (100), 115 (2.56), 105 (4.46), 102 (48.54), 75 (12.81), 51 (9.83), 42 (6.46); $\mathrm{C}_{19} \mathrm{H}_{17} \mathrm{NO}_{2}$ Si requires C, 71.44; H, 5.36; N, 4.38; found: $\mathrm{C}, 71.19 ; \mathrm{H}, 5.19 ; \mathrm{N}, 4.50$. 
4'-(2-Trimethylsilylethynyl)phenyl-4-nitrobenzoate (3b). IR: 2922, 2854, 1745, 1527 , 1498, 1456, 1348, 1261, 1201, 1078, 1014, $850 \mathrm{~cm}^{-1} ;{ }^{1} \mathrm{H}$ NMR (ppm, $\mathrm{CDCl}_{3}$ ): 8.3 (s, $4 \mathrm{H}), 7.5(\mathrm{~d}, 2 \mathrm{H}, J=8.8 \mathrm{~Hz}), 7.2(\mathrm{~d}, 2 \mathrm{H}, J=8.8 \mathrm{~Hz}), 0.26(\mathrm{~s}, 9 \mathrm{H}) ;{ }^{13} \mathrm{C}$ NMR $(\mathrm{ppm}$, $\left.\mathrm{CDCl}_{3}\right)$ : 162.9, 151, 150.4, 134.7, 133.3, 131.3, 123.8, 121.5, 121.4, 103.9, 94.9, 0.02; GC-MS: 339 (m/e, relative intensity), 207 (1.43), 193 (0.26), 150 (19.08), 146 (1.5), 134 (2.75), 120 (2.10), 104 (11.77), 76 (11.92), 73 (11.46), 63 (1.58), 55 (7.05), 44 (64.42), 40 (100); $\mathrm{C}_{18} \mathrm{H}_{17} \mathrm{NO}_{4} \mathrm{Si}$ requires C, 63.70; H, 5.05; N, 4.13; found: C, 63.86; H, 4.99; N, 4.17 .

4'-(2-Trimethylsilylethynyl)phenyl-3-methoxybenzoate (3c). IR: 2960, 2837, 2158, 1730, 1600, 1504, 1465, 1276, 1199, 1039, 908, $746 \mathrm{~cm}^{-1} ;{ }^{1} \mathrm{H}$ NMR (ppm, $\left.\mathrm{CDCl}_{3}\right): 7.7$ $(\mathrm{m}, 1 \mathrm{H}), 7.6(\mathrm{~m}, 1 \mathrm{H}), 7.5(\mathrm{~d}, 2 \mathrm{H}, J=2.0 \mathrm{~Hz}), 7.4(\mathrm{t}, 1 \mathrm{H}, J=8.0 \mathrm{~Hz}), 7.1(\mathrm{~m}, 3 \mathrm{H}), 3.9$ (s, 3H), 0.26 (s, 9H); ${ }^{13} \mathrm{C}$ NMR (ppm, $\mathrm{CDCl}_{3}$ ): 163.2, 150.4, 133.3, 133.1, 132.4, 130.6, 121.4, 117.7, 117.1, 103, 94.8, 0.02; GC-MS: 324 (m/e, relative intensity), 309 (0.55), 174 (2.67), 146 (4.43), 136 (8.25), 135 (100), 107 (20.88), 92 (14.73), 77 (25.86), 64 (7.60), 63 (6.37), 53 (2.77), 50 (2.59), 43 (3.19); $\mathrm{C}_{19} \mathrm{H}_{20} \mathrm{O}_{3}$ Si requires C, 70.34; H, 6.21; found: $\mathrm{C}$, $70.38 ; \mathrm{H}, 6.10$.

4'-(2-Trimethylsilylethynyl)phenyl-4-benzyloxybenzoate (3d). IR: 2922, 2852, 2162, 1724, 1606, 1462, 1280, 1074, 987, $744 \mathrm{~cm}^{-1} ;{ }^{1} \mathrm{H}$ NMR (ppm, $\left.\mathrm{CDCl}_{3}\right): 8.1$ (d, $2 \mathrm{H}, J=$ $9.0 \mathrm{~Hz}), 7.5(\mathrm{~d}, 2 \mathrm{H}, J=8.8 \mathrm{~Hz}), 7.4(\mathrm{~m}, 5 \mathrm{H}), 7.1(\mathrm{~d}, 2 \mathrm{H}, J=8.8 \mathrm{~Hz}), 7.0(\mathrm{~d}, 2 \mathrm{H}, J=$ $9.0 \mathrm{~Hz}), 5.1(\mathrm{~s}, 2 \mathrm{H}), 0.26(\mathrm{~s}, 9 \mathrm{H}) ; \mathrm{C}_{25} \mathrm{H}_{24} \mathrm{O}_{3} \mathrm{Si}$ requires $\mathrm{C}, 74.97$; $\mathrm{H}, 6.04$; found: $\mathrm{C}, 75.03$; H, 6.18 .

4'-(2-Trimethylsilylethynyl)phenyl-3,4,5-tris(n-hexyloxy)benzoate (3e). IR: 2929, 2856, 2119, 1735, 1585, 1502, 1452, 1338, 1247, 1188, 1045, $842 \mathrm{~cm}^{-1} ;{ }^{1} \mathrm{H}$ NMR (ppm, $\left.\mathrm{CDCl}_{3}\right): 7.5(\mathrm{~d}, 2 \mathrm{H}, J=8.8 \mathrm{~Hz}), 7.4(\mathrm{~d}, 2 \mathrm{H}, J=2.6 \mathrm{~Hz}), 7.1(\mathrm{~d}, 2 \mathrm{H}, J=8.7 \mathrm{~Hz}), 4.0(\mathrm{~m}$, $6 \mathrm{H}), 1.8-1.2(\mathrm{~m}, 24 \mathrm{H}), 0.9(\mathrm{~m}, 9 \mathrm{H}), 0.26(\mathrm{~s}, 9 \mathrm{H}) ; \mathrm{C}_{36} \mathrm{H}_{54} \mathrm{O}_{5} \mathrm{Si}$ requires $\mathrm{C}, 72.68 ; \mathrm{H}, 9.15$; found: $\mathrm{C}, 72.51 ; \mathrm{H}, 9.21$.

4'-(2-Trimethylsilylethynyl)phenyl-3,4-bis(n-decyloxy)benzoate (3f). IR: 2920, 2852, 2152, 1735, 1597, 1462, 1377, 1273, 1138, 1018, 842, $754 \mathrm{~cm}^{-1} ;{ }^{1} \mathrm{H}$ NMR (ppm, $\mathrm{CDCl}_{3}$ ): $7.8(\mathrm{~d}, 1 \mathrm{H}, J=2.0 \mathrm{~Hz}), 7.6(\mathrm{~d}, 1 \mathrm{H}, J=2.0 \mathrm{~Hz}), 7.5(\mathrm{~d}, 2 \mathrm{H}, J=8.8 \mathrm{~Hz}), 7.1(\mathrm{~d}, 2 \mathrm{H}, J=$ $1.8 \mathrm{~Hz}), 6.9(\mathrm{~d}, 1 \mathrm{H}, J=8.8 \mathrm{~Hz}), 4.2(\mathrm{t}, 4 \mathrm{H}, J=6.5 \mathrm{~Hz}), 1.8(\mathrm{~m}, 4 \mathrm{H}), 1.6-1.2(\mathrm{~m}, 28 \mathrm{H})$, $0.9(\mathrm{tt}, 6 \mathrm{H}, J=3.1 \mathrm{~Hz}$ and $3.0 \mathrm{~Hz}), 0.25(\mathrm{~s}, 9 \mathrm{H}) ; \mathrm{C}_{38} \mathrm{H}_{58} \mathrm{O}_{4} \mathrm{Si}$ requires $\mathrm{C}, 75.20 ; \mathrm{H}, 9.63$; found: C, 75.11; H, 9.59.

4'-(2-Trimethylsilylethynyl)phenyl-4-n-heptyloxybenzoate (3g). [5] IR: 2906, 2854, 2160, 1741, 1608, 1510, 1454, 1377, 1251, 1201, 1165, 1066, $842 \mathrm{~cm}^{-1} ;{ }^{1} \mathrm{H}$ NMR (ppm, $\left.\mathrm{CDCl}_{3}\right): 8.1(\mathrm{~d}, 2 \mathrm{H}, J=2.0 \mathrm{~Hz}), 7.5(\mathrm{~d}, 2 \mathrm{H}, J=1.9 \mathrm{~Hz}), 7.1(\mathrm{~d}, 2 \mathrm{H}, J=1.8 \mathrm{~Hz})$, $6.9(\mathrm{~d}, 2 \mathrm{H}, J=1.7 \mathrm{~Hz}), 4.0(\mathrm{t}, 2 \mathrm{H}, J=6.5 \mathrm{~Hz}), 1.8-1.2(\mathrm{~m}, 10 \mathrm{H}), 0.9(\mathrm{t}, 3 \mathrm{H}, J$ $=6.7 \mathrm{~Hz}), 0.25(\mathrm{~s}, 9 \mathrm{H}) ; \mathrm{C}_{25} \mathrm{H}_{32} \mathrm{O}_{3} \mathrm{Si}$ requires $\mathrm{C}, 73.49 ; \mathrm{H}, 7.89$; found: $\mathrm{C}, 73.58 ; \mathrm{H}$, 8.01 .

4'-(2-Trimethylsilylethynyl)phenyl-4-n-tetradecyloxy)benzoate (3h). [5] IR: 2924, 2854, 2158, 1732, 1606, 1508, 1458, 1251, 1165, 1068, $842 \mathrm{~cm}^{-1} ;{ }^{1} \mathrm{H} \mathrm{NMR}$ (ppm, $\mathrm{CDCl}_{3}$ ): $8.1(\mathrm{~d}, 2 \mathrm{H}, J=2.0 \mathrm{~Hz}), 7.5(\mathrm{~d}, 2 \mathrm{H}, J=1.9 \mathrm{~Hz}), 7.1(\mathrm{~d}, 2 \mathrm{H}, J=1.8 \mathrm{~Hz}), 6.9(\mathrm{~d}, 2 \mathrm{H}, J=$ $1.8 \mathrm{~Hz}), 4.0(\mathrm{t}, 2 \mathrm{H}, J=6.5 \mathrm{~Hz}), 1.8-1.2(\mathrm{~m}, 24 \mathrm{H}), 0.9(\mathrm{t}, 3 \mathrm{H}, J=6.6 \mathrm{~Hz}), 0.25(\mathrm{~s}, 9 \mathrm{H})$; $\mathrm{C}_{32} \mathrm{H}_{46} \mathrm{O}_{3}$ Si requires $\mathrm{C}$, 75.84; $\mathrm{H}, 9.15$; found: C, 76.01; H, 9.08 .

4'-(n-Dodecyl-biphenyl-4-carboxylic-(2-trimethylsilylethynyl)phenyl ester (3i). IR: 2922, 2852, 2162, 1730, 1606, 1502, 1454, 1377, 1247, 1203, 1080, $840 \mathrm{~cm}^{-1} ;{ }^{1} \mathrm{H}$ NMR $\left(\mathrm{ppm}, \mathrm{CDCl}_{3}\right): 8.2(\mathrm{~d}, 2 \mathrm{H}, J=1.9 \mathrm{~Hz}), 7.7(\mathrm{~d}, 2 \mathrm{H}, J=2.0 \mathrm{~Hz}), 7.5(\mathrm{~m}, 4 \mathrm{H}), 7.3(\mathrm{~d}, 2 \mathrm{H}$, $J=8.1 \mathrm{~Hz}), 7.1(\mathrm{~d}, 2 \mathrm{H}, J=2.0 \mathrm{~Hz}), 2.6(\mathrm{t}, 2 \mathrm{H}, J=7.5 \mathrm{~Hz}), 1.6-1.2(\mathrm{~m}, 20 \mathrm{H}), 0.9(\mathrm{t}$, $3 \mathrm{H}, J=6.8 \mathrm{~Hz}), 0.26(\mathrm{~s}, 9 \mathrm{H}) ; \mathrm{C}_{36} \mathrm{H}_{50} \mathrm{O}_{2}$ Si requires $\mathrm{C}, 79.65 ; \mathrm{H}, 9.28$; found: $\mathrm{C}, 79.80 ; \mathrm{H}$, 9.31. 
4-(4'-n-Hexadecyloxybenzoyloxy)carboxylic-4-trimethylsilylethynyl-phenyl ester (3j). IR: 2922, 2852, 2158, 1738, 1608, 1512, 1462, 1377, 1282, 1166, 1018, $846 \mathrm{~cm}^{-1}$; ${ }^{1} \mathrm{H}$ NMR (ppm, $\left.\mathrm{CDCl}_{3}\right): 8.2(\mathrm{dd}, 2 \mathrm{H}, J=2.0 \mathrm{~Hz} \& 8.8 \mathrm{~Hz}), 8.1(\mathrm{~d}, 2 \mathrm{H}, J=8.8 \mathrm{~Hz}), 7.7$ $(\mathrm{d}, 1 \mathrm{H}, J=8.8 \mathrm{~Hz}), 7.5(\mathrm{~d}, 1 \mathrm{H}, J=8.8 \mathrm{~Hz}), 7.3(\mathrm{~d}, 2 \mathrm{H}, J=8.5 \mathrm{~Hz}), 7.1(\mathrm{~d}, 1 \mathrm{H}, J=$ $8.7 \mathrm{~Hz}), 7.0(\mathrm{~m}, 3 \mathrm{H}), 4.0(\mathrm{t}, 2 \mathrm{H}, J=6.5 \mathrm{~Hz}), 1.8-1.2(\mathrm{~m}, 28 \mathrm{H}), 0.9(\mathrm{t}, 3 \mathrm{H}, J=6.6 \mathrm{~Hz})$, $0.26(\mathrm{~s}, 9 \mathrm{H}) ; \mathrm{C}_{41} \mathrm{H}_{54} \mathrm{O}_{5} \mathrm{Si}$ requires $\mathrm{C}, 75.19 ; \mathrm{H}, 8.31$; found: $\mathrm{C}, 75.23 ; \mathrm{H}, 8.52$.

Preparation of 1-(4'-n-Pentyloxyphenyl)-2-trimethylsilylacetylene (5). [4,10] A mixture of 1-iodo-4-(pentyloxy)benzene $(2 \mathrm{~g}, 0.34 \mathrm{mmol})$, copper acetate monohydrate $(1.2 \mathrm{~g}$, $0.85 \mathrm{mmol})$, and palladium chloride $(1.6 \mathrm{~g}, 0.85 \mathrm{mmol})$ in triethylamine $(5 \mathrm{~mL})$ was stirred in nitrogen atmosphere at room temperature. Trimethylsilylacetylene $(0.76 \mathrm{~g}, 0.40 \mathrm{mmol})$ was added dropwise over $30 \mathrm{~min}$ and after completion of addition the reaction mixture refluxed at $80^{\circ} \mathrm{C}$ overnight. The reaction was monitored by TLC. After completion of reaction, the mixture was cooled and filtered. The volatile solvents were removed under reduced pressure. The resulting crude light brown colored viscous oil was extracted into ether, washed with saturated potassium bicarbonate solution, dried over anhydrous sodium sulfate, and concentrated. Finally, the silylated product was purified by column chromatography on silica gel with 8:2 ratio of hexane-ethylacetate as an eluent. Yield $=1.26 \mathrm{~g}$ $(73 \%)$.

Preparation of 1-Ethynyl-4'-(n-pentyloxy)benzene (6) [12]. To the 1-(4'-n-pentyloxyphenyl)-2-trimethylsilylacetylene $(1 \mathrm{~g}, 0.38 \mathrm{mmol})$ was added potassium carbonate $(1.0 \mathrm{~g}, 0.76 \mathrm{mmol})$ in cold methanol at ambient temperature. The resultant reaction mixture was stirred for $4 \mathrm{~h}$ under inert atmosphere. The reaction was monitored by TLC. After complete conversion of starting material into product, the reaction mixture was filtered. The volatile solvents were removed under reduced pressure. The resulting crude light brown colored viscous residue was extracted into ether, washed with saturated potassium bicarbonate solution, dried over anhydrous sodium sulfate, and concentrated. The crude product was purified by column chromatography on silica gel using 2:8 ratio of ethyl acetate and hexane as mobile phase. Viscous liquid, Yield $=0.530 \mathrm{~g}, 76 \%$.

1,4-Bis[4'-(n-pentyloxy)phenyl]buta-1,3-diyne (7). [12,13] To a solution of dry pyridine $(5 \mathrm{~mL})$ and methanol $(5 \mathrm{~mL})$ was added 1-ethynyl-4-(pentyloxy)benzene $(0.250 \mathrm{~g}$, $1 \mathrm{mmol})$ and copper acetate $(0.601 \mathrm{~g}, 2.5 \mathrm{mmol})$ under argon atmosphere. The resultant reaction mixture was refluxed at $80^{\circ} \mathrm{C}$ for $24 \mathrm{~h}$. The reaction mixture was cooled, filtered, and solvents were removed under reduced pressure. The crude brown colored product was purified by column chromatography on silica gel using $10 \%$ ethyl acetate in petroleum ether. The product was crystallized in chloroform as colorless crystal; IR: 2916, 2856, 2119 , 1735, 1585, 1502, 1431, 1338, 1247, 1188, 1045, $842 \mathrm{~cm}^{-1} ;{ }^{1} \mathrm{H}$ NMR (ppm, $\mathrm{CDCl}_{3}$ ): 7.43 $(\mathrm{d}, 4 \mathrm{H}, J=8.7 \mathrm{~Hz}), 6.83(\mathrm{~d}, 4 \mathrm{H}, J=8.7 \mathrm{~Hz}), 3.95(\mathrm{t}, 4 \mathrm{H}, J=6.5 \mathrm{~Hz}), 1.8-1.40(\mathrm{~m}, 12 \mathrm{H})$, $0.93(\mathrm{t}, 6 \mathrm{H}, J=4.5 \mathrm{~Hz}) ;{ }^{13} \mathrm{C} \mathrm{NMR}\left(\mathrm{ppm}, \mathrm{CDCl}_{3}\right)$ : 159.8, 134, 114.6, 113.7, 81.3, 72.9, 68.21, 28.3, 28.1, 22.42, 13.9; $\mathrm{C}_{26} \mathrm{H}_{30} \mathrm{O}_{2}$ requires $\mathrm{C}$, 83.38; $\mathrm{H}, 8.07$; found: $\mathrm{C}, 82.29 ; \mathrm{H}$, 7.92.

1,4-Bis[4'(n-pentyloxy)phenyl]butane (8). A mixture of compound 7 (0.2 g, $1 \mathrm{mmol})$ dissolved in ethyl acetate $(20 \mathrm{~mL})$ and $5 \% \mathrm{Pd}-\mathrm{C}$ catalyst $(0.2 \mathrm{~g}, 3 \mathrm{mmol})$ was stirred in an atmosphere of hydrogen for $12 \mathrm{~h}$. The resulting mixture was filtered and the solvent removed under reduced pressure. The solid material obtained was recrystalized from ethanol. Yield: 0.172 g; Mp: $75-77^{\circ} \mathrm{C}$; IR: 2922, 2852, 1654, 1456, 1375, 1244, 1174, $1018 \mathrm{~cm}^{-1} ;{ }^{1} \mathrm{H}$ NMR (ppm, $\left.\mathrm{CDCl}_{3}\right): 7.06(\mathrm{~d}, 4 \mathrm{H}, J=8.8 \mathrm{~Hz}), 6.81(\mathrm{~d}, 4 \mathrm{H}, J=8.8 \mathrm{~Hz}), 3.94(\mathrm{t}, 4 \mathrm{H}, J=$ $6.5 \mathrm{~Hz}), 2.8(\mathrm{~s}, 4 \mathrm{H}), 1.79-1.25(\mathrm{~m}, 16 \mathrm{H}), 0.94(\mathrm{t}, 6 \mathrm{H}, J=4.6 \mathrm{~Hz}) ; \mathrm{C}_{26} \mathrm{H}_{39} \mathrm{O}_{2}$ requires $\mathrm{C}$, 81.62; H, 10.01; found: C, 81.53, H, 10.22 . 
Table 2. Transition temperatures and enthalpies $\left(\Delta H \mathrm{~kJ} / \mathrm{mol}^{-1}\right)$ of compounds $\mathbf{3 i}$ and $\mathbf{3 j}$ and 7; heating/cooling scan rates at $5^{\circ} \mathrm{C}$ min.

\begin{tabular}{lcc}
\hline Compound & Heating Scan $\left({ }^{\circ} \mathrm{C}\right)$ & Cooling Scan $\left({ }^{\circ} \mathrm{C}\right)$ \\
\hline $\mathbf{3 i}^{\mathrm{a}}$ & Cr 90 SmA 160 Iso & Iso 157 SmA 70 Cr \\
$\mathbf{3 j}^{\mathrm{a}}$ & Cr 102 SmA 176 Iso & Iso 173 SmA 80 Cr \\
$\mathbf{7}$ & Cr 120.3(89.2) N 157.3(4.35) Iso & Iso 156.5(4.56) N 116.9(85.65) Cr \\
\hline
\end{tabular}

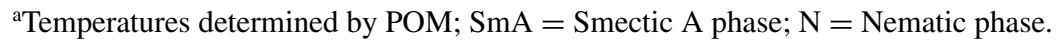

\section{Results and Discussion}

\section{Synthesis}

1-(4'-Hydroxyphenyl)-2-trimethylsilylacetylene (2) was used to prepare rod-shaped molecules $\mathbf{3 a - j}$ by simple and efficient DCC coupling with various substituted benzoic acids according to the general procedure given in the experimental section. Our procedure simplifies laborious synthesis of compound $\mathbf{2}$ to get a range of compounds with variation of the terminal unit. All compounds that we have synthesized constituted of polar terminal substituents like cyano-, nitro-, groups with two aryl ring compounds as indicated in 3a-j.

In further studies, we prepared the dialkoxydiphenyldiacetylene compound 7 . We have converted 4-iodophenol 1 into 4-(pentyloxy)iodobenzene 4 by Williamson etherification using appropriate quantity of 1-bromopentane. Subsequently, 1-(4'-n-pentyloxyphenyl)-2trimethylsilylacetylene was prepared by palladium-catalyzed Sonogashira coupling, followed by basic hydrolysis using potassium carbonate and methanol as a proton source to get 1-ethynyl-4-(pentyloxy)benzene 6 (Scheme 1). Compound 6 was used for Sonogashira homo-coupling reaction to obtain compound 7 , which was subjected to reduction with $5 \%$ palladium on charcoal and hydrogen gas at atmospheric pressure and room temperature to get $\mathbf{8}$ with flexible $n$-butyl spacer. The chemical structures were confirmed by comparison with the corresponding literature $[12,13]$.

The compounds $\mathbf{3} \mathbf{a}-\mathbf{j}$ were evaluated for the liquid crystalline properties using DSC and POM techniques. Compounds $\mathbf{3 a}$ and $\mathbf{3 b}$ did not exhibit liquid crystalline property. The compounds with methoxy- 3c, benzyloxy- 3d, tri- $n$-hexyloxy- 3e, and di- $n$-decyloxy- $\mathbf{3 f}$ moieties also hardly showed any liquid crystalline property. This prompted us to increase the substituent by one more aryl ring to verify the structure-property relationships of the liquid crystals.

In this regard, we synthesized the 2-trimethylsilylacetylene group containing known monotropic SmA phase compound $\mathbf{3 g}$ and nonmesogenic compound $\mathbf{3 h}$ [11]. These compounds $\mathbf{3 g}$ and $\mathbf{3 h}$ and their respective acetylenes show thermodynamically unstable mesophases, probably due to steric hindrance of the much larger trimethylsilyl group present in the terminal position of the molecule.

To overcome this, and to achieve thermodynamically stable mesophase, we prepared the compounds $\mathbf{3 i}$ and $\mathbf{3 j}$ by increasing the length of the molecule with one phenyl ring and a single long alkyl chain. Compound 3i, a biphenyl derivative without connecting group; and $\mathbf{3 j}$ with ester connecting group possess long alkyl chains which favor the mesomorphic property. 


\section{Liquid Crystal Property}

Melting and isotropic transition points, which are liquid crystalline behaviors, were deduced by the combination of DSC and polarized optical microscopic observations. Liquid crystal phase transition temperatures are shown in Table 2.

The compounds $3 \mathbf{i}$ and $\mathbf{3 j}$ show a fan-shaped texture of Smectic A phase enantiotropically (Fig. 1(a)). Compound $\mathbf{3 i}$ has lower mesophase stability and width of the mesophase than that of the molecule $\mathbf{3 j}$. The ester linkage of $\mathbf{3 j}$ gives additional stability in relation to the physical properties such as mesophase range and melting and isotropic temperatures.

a)

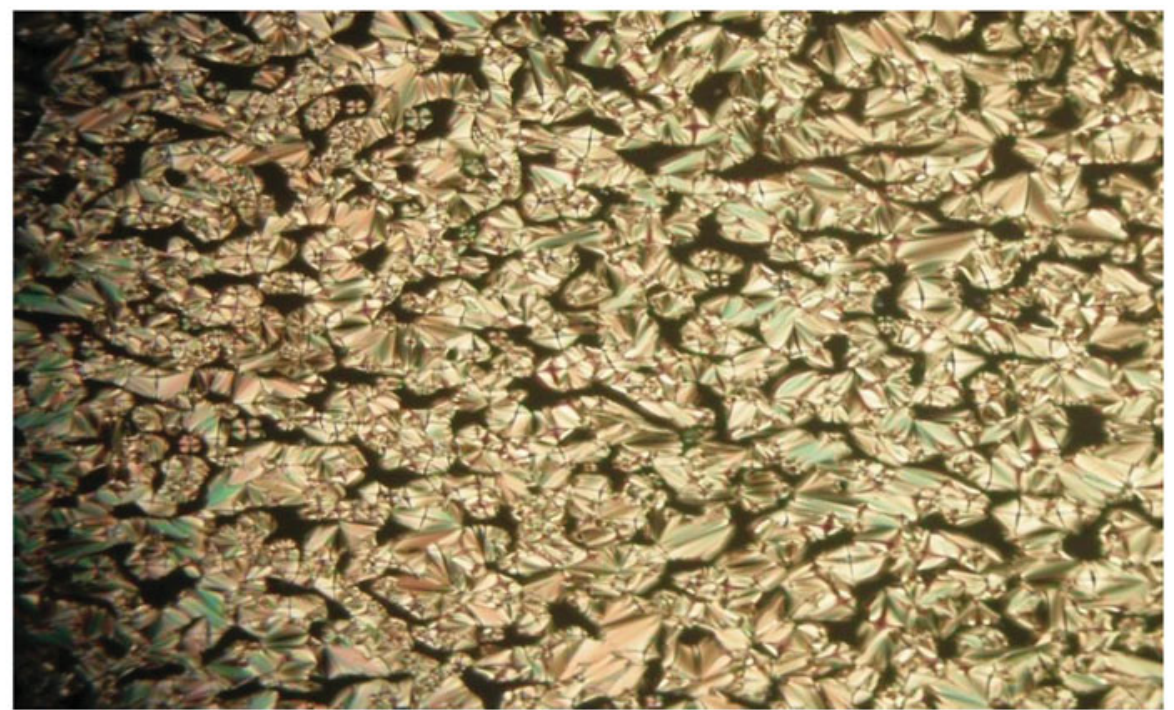

b)

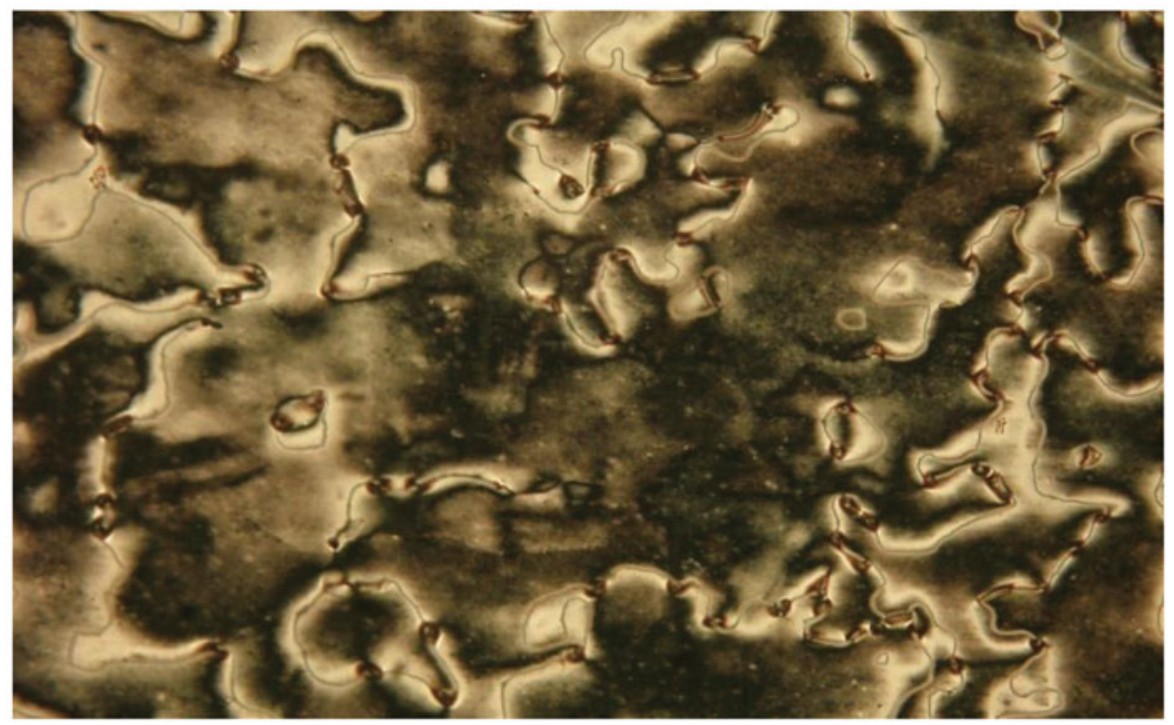

Figure 1. Optical photomicrographs: a) compound 3j Smectic A phase at $125^{\circ} \mathrm{C}$ and b) compound 7 at $140^{\circ} \mathrm{C}$ Nematic phase obtained on cooling from isotropic states. 


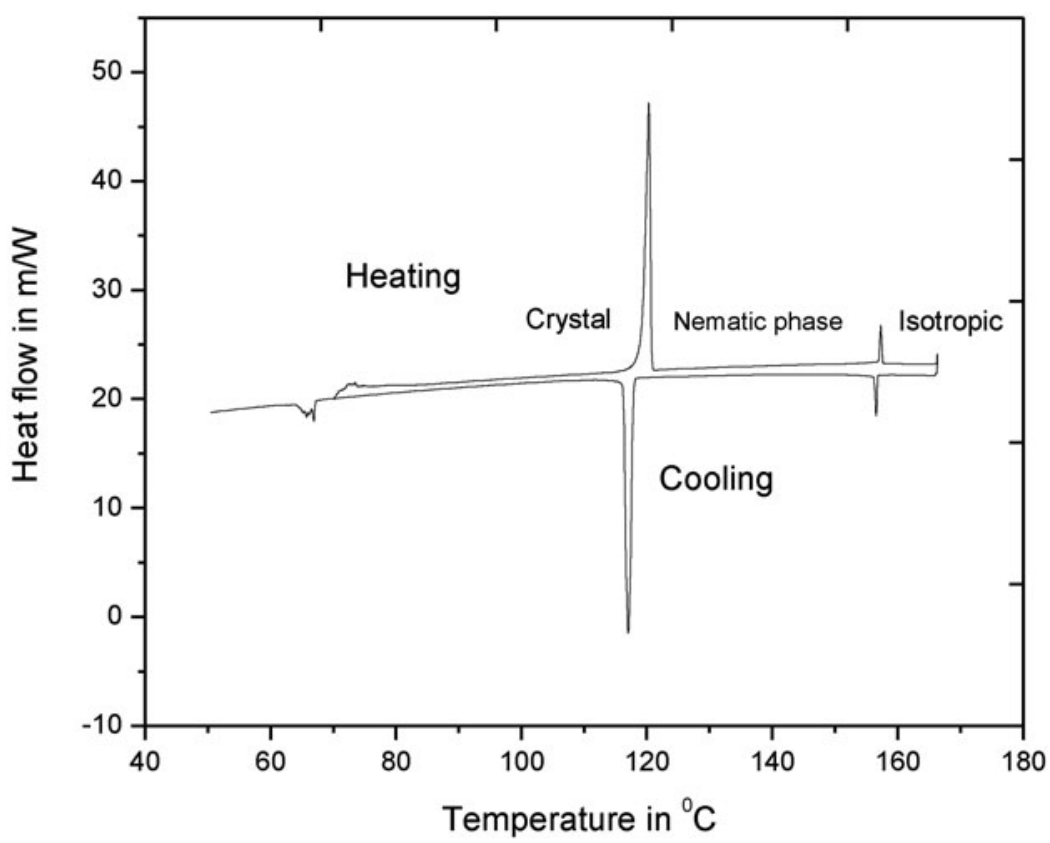

Figure 2. A DSC thermogram obtained for compound 7 showing phase transition; heating and cooling cycles scan rate at $5^{\circ} \mathrm{C} / \mathrm{min}$.

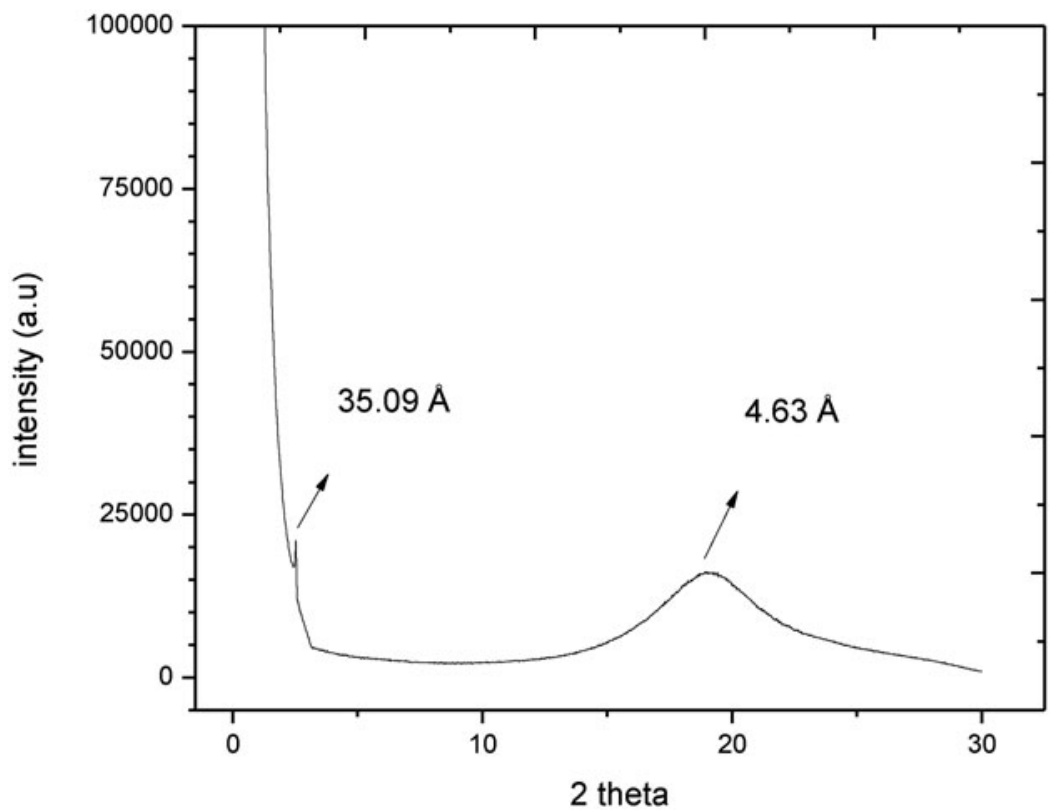

Figure 3. X-ray diffractogram of compound $\mathbf{3 i}$ in the mesophase at $110^{\circ} \mathrm{C}$. 


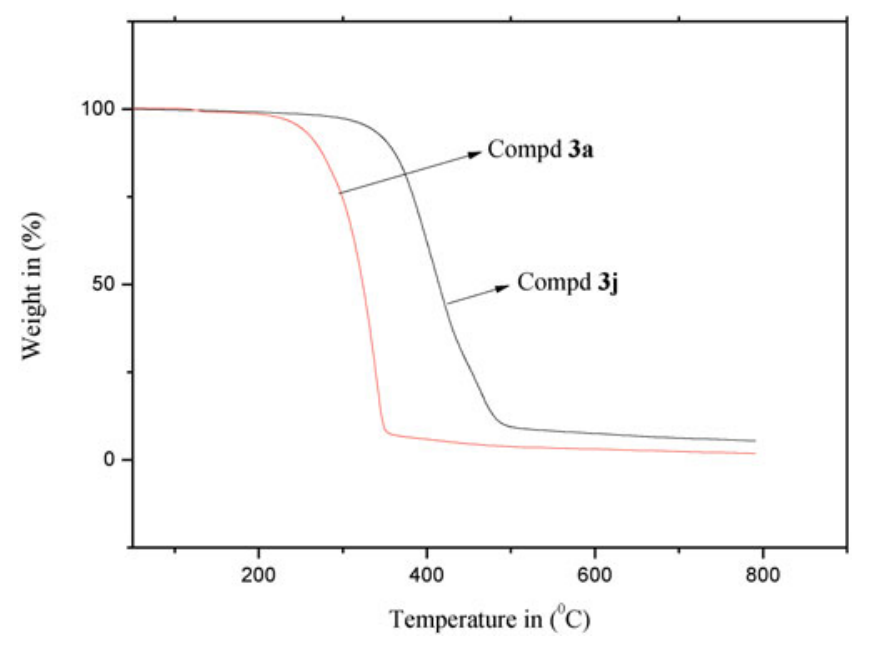

Figure 4. Representative TGA curves of compounds $\mathbf{3 a}$ and $\mathbf{3 j}$.

Compound 7 shows a long-range Nematic mesophase (Fig. 1(b)) at about $40^{\circ} \mathrm{C}$, which melts from crystalline to liquid crystalline state at $120.3{ }^{\circ} \mathrm{C}$ (89.25) (Fig. 2), and then it goes into isotropic state at $157.3^{\circ} \mathrm{C}$ (4.35). Upon cooling from isotropic liquid, a peculiar Schleiren texture of Nematic phase appears at $156.5^{\circ} \mathrm{C}(-4.56)$. The material crystallizes at $116.9^{\circ} \mathrm{C}(-85.65)$.

The reduction and formation of $\mathbf{8}$ too did not show liquid crystalline property. This is due to the introduction of flexibility at the center of the low molar mass molecules leading to disruption of molecular packing and therefore, loss of the liquid crystalline property.

X-ray analysis was carried out for the compound $\mathbf{3 i}$ for further confirmation of the mesophase. The intensity versus $2 \theta$ plot was derived from the diffraction pattern as shown in Fig. 3. In the small angle region, a sharp peak corresponding to spacing of $35.09 \AA$ and in the wide angle region a spacing of $4.63 \AA$ were indicative of liquid-like order, local molecular structure, and typical scattering pattern, thus confirming the SmA mesophase.

The thermal stability of compounds $\mathbf{3 a}$ and $\mathbf{3 j}$ was studied by ThermoGravimetric Analysis, with the representative TGA curves shown in Fig. 4. According to TGA analysis, compounds $\mathbf{3 a}$ and $\mathbf{3 j}$ have good thermal stability up to $200-250^{\circ} \mathrm{C}$ and temperatures at which $100 \%$ weight loss occurred were between $210^{\circ} \mathrm{C}$ and $500^{\circ} \mathrm{C}$.

This study illustrated the effect of highly polar and nonpolar alkoxy substituents in the molecular structure on the liquid crystal property of rod-shaped molecules derived from aryl 2-(trimethylsilyl)ethynyl. We observed that the polar substituent at the terminal position does not favor mesomorphism in the low molecular weight compounds possessing an ethynyltrimethylsilyl group.

\section{Conclusions}

Ten novel 4-trimethylsilylethynyl group substituted benzoates and aryloxydiacetylene rodshaped molecules have been synthesized with simple and efficient coupling procedures. All chemical structures have been confirmed by standard spectroscopic techniques. The bulky 
and tetrahedron geometry of the trimethylsilyl group does not favor the mesomorphic property without elongation of the molecular length. On the other hand, only terminal monoalkylated extended phenyl ring compounds show mesomorphic property.

\section{Acknowledgments}

We are thankful to Mrs. K. N. Vasudha, RRI, Bengaluru, for thermal and X-ray analysis. Grateful thanks to the NMR Research Center, Indian Institute of Science, for NMR spectral analysis. Financial support by the University Grants Commission and Department of Science and Technology, New Delhi, India, is gratefully acknowledged.

\section{References}

[1] Yamanishi, H., Tomita, I., Ohta, K., \& Endo. T. (2001). Mol. Cryst. Liq. Cryst., 369, 47.

[2] Celanire, S., Marlin, F., Baldwin, J. E., \& Adlington, R. M. (2005). Tetrahedron, 61, 3025.

[3] Schafer, C., Herrmann, F., \& Mattay. J. (2008). Beilstein. J. Org. Chem., 4, 1.

[4] Kaharu, T., Matsubara, H., \& Takahashi. S. (1992). J. Mater. Chem., 2, 43.

[5] Ho, M. S., \& Hsu, C. S. (2010). Liq. Cryst., 37, 293.

[6] Radini, I. A., \& Hird, M. (2009). Liq. Cryst., 36, 1417.

[7] Hudson, C. M., Shenoy, R. A., Neubert, M. E., Brown, G. H., \& Petschek, R. G. (1999). Liq. Cryst., 26, 241.

[8] Mohamed Ahmed, M. S., \& Mori. A. (2004). Tetrahedron, 60, 9977.

[9] Skelton, G. W., Dong, D., Tuffin, R. P., \& Kelly, S. M. (2003). J. Mater. Chem., 13, 450.

[10] Lydon, D. P., Albesa-Jove, D., Shearman, G. C., Seddon, J. M., Howard, J. A. K., Mardera, T. B., \& Low, P. J. (2008). Liq. Cryst., 35, 119.

[11] Hsu, H. F., Lai, Y. H., Lin, S Y., Lin, W. C., \& Chenl, J. F. (2003). Liq. Cryst., $30,325$.

[12] Arakawa, Y., Nakajima, S., Ishige, R., Uchimura, M., Kang, S., Konishi, G., \& Watanabe, J. (2012). J. Mater. Chem., 22, 8394.

[13] Yatabe, T., \& Kawanishi, Y. (2011). Liq. Cryst., 39, 1.

[14] Pugh, C., \& Percec. V. (1999). Mol. Cryst. Liq. Cryst., 178, 193.

[15] Lee, C. H., Jeong, N. H., Nam, K. D., \& Kitamura. T. (1999). J. Ind \& Eng. Chem., 5, 40.

[16] Long, T. M., \& Swager, T. M. (2002). J. Mater. Chem., 12, 3407.

[17] Hennrich, G., Ortiz, P. D., Cavero, E., Hanes, R. E., \& Serrano, J. L. (2008). Eur. J. Org. Chem., 4575 .

[18] Demus, D., Goodby, G., Gray, G. W., Spiess, H. W., \& V. Vill. (1998). Hand Book of Liquid Crystals, vols. 1-3, Wiley-VCH: Weinhem.

[19] Yashima, E., Huang, S., Matsushima, T., \& Okamoto. Y. (1995). Macromolecules, 28, 4184.

[20] Hanasaki, T., Kamei, Y., Mandai, A., Uno, K., \& Kaneko, K. (2011). Liq. Cryst., 38, 841.

[21] Liao, C. T., Wu, Z. L., Wu, N. C., Liu, Z. Y., Jiang, M. H., Zou, S. F., \& Lee, J. Y. (2010). Mol. Cryst. Liq. Cryst., 533, 3.

[22] Srinivasa, H. T., \& Hariprasad, S. (2012). Org. Commun., 5(3), 128.

[23] Srinivasa, H. T., \& Hariprasad, S. (2012). Org. Chem. Ind. J., 8, 130.

[24] Divya Jyothi, \& Hariprasad, S. (2012). ARKIVOC, 6, 194.

[25] Hariprasad, S., \& Nagendrappa, G. (1993). Tetrahedron, 49, 3387.

[26] Hariprasad, S., \& Nagendrappa, G. (1997). Indian J. Chem., Sect. B: Org. Chem. Incl. Med. Chem., 36, 1016.

[27] Venkatesha, M. A., \& Hariprasad, S. (2012). J. Bulg. Chem. Soc., 44, 155.

[28] Naciri, J., Carboni, C., \& Georgel, A. K. (2003). Liq. Cryst., 30, 219.

[29] Radhika, S., Srinivasa, H. T., \& Sadashiva, B. K. (2011). Liq. Cryst., 38, 785.

[30] Cowling, S. J., Hall, A. W., \& Goodby, J. W. (2011). J. Mater. Chem., 21, 9031. 